\title{
Rechtsprechung: Werbung für Sexualkontakte durch Prostituierte
}

Martin Schaar

\section{Leitsätze:}

UWG $\S 2$ Abs. 1 Nr. 3, $\S 33,4$ Nr. 11, $\S 8$ Abs. 3 Nr. 1

OWiG § 119 Abs. 1, § 120 Abs. 1 Nr. 2

a) Zwischen Prostituierten und dem Betreiber einer Bar, in denen Prostituierten und deren Kunden sexuelle Kontakte ermöglicht werden, besteht ein unmittelbares Wettbewerbsverhältnis.

b) Das Verbot der Werbung für Prostitution nach $\S 119$ Abs. 1, § 120 Abs. 1 Nr. 2 OWiG ist auch dazu bestimmt, im Interesse von Marktteilnehmern das Marktverhalten zu regeln ( $§ 4$ Nr. 11 UWG).

c) Ein Werbeverbot nach $\S 120$ Abs. $1 \mathrm{Nr}$. 2 OWiG setzt die konkrete Eignung der Werbung voraus, den Schutz der Allgemeinheit, vor allem von Kindern und Jugendlichen, vor den mit der Prostitution generell verbundenen Gefahren und Belästigungen zu beeinträchtigen.

BGH, Urt. v. 13. Juli 2006 - I ZR 241/03, I ZR 231/03, I ZR 65/05 - jeweils

OLG Hamm, LG Bielefeld

\section{Sachverhalt:}

Den Urteilen lag im Wesentlichen der gleiche Sachverhalt zugrunde: Die Kläger betreiben eine Bar, in der Prostituierten und deren Kunden sexuelle Kontakte ermöglicht werden. Die Beklagten waren jeweils Herausgeberinnen von Zeitungen, in denen auch Inserate mit Werbung für sexuelle Kontakte veröffentlicht wurden. Hiergegen machten die Kläger einen Unterlassungsanspruch nach $\S 8$ Abs. 1 S. 1, §§ 3, 4 Nr. 11 UWG i.V. mit $\S 119$ Abs. 1 , § 120 Abs. 1 Nr. 2 OWiG geltend, da die Inserate unzulässige Werbung für Prostitution darstellen würden.

Das Landgericht hatte die Klagen abgewiesen und das Berufungsgericht die Urteile bestätigt. Die Revision blieb ohne Erfolg.

\section{Anmerkung:}

Die Urteile, die auf den ersten Blick hauptsächlich das Wettbewerbsrecht zum Gegenstand haben, bieten bei näherem Hinschauen Anlass, sich mit der Umsetzung des ProstG ${ }^{1}$ beziehungsweise dessen Ausstrahlungswirkung auf andere Rechtsgebiete auseinanderzusetzen. Denn der BGH stellte zwar, anders als das Landgericht und das Berufungsgericht fest, dass zwischen den Klägern und den Anzeigenkunden der Beklagten ein Wettbewerbsverhältnis bestehe und dass das Verbot der Werbung für Prostitution nach §§ 119 Abs. 1, 120 Abs. 1 Nr. 1 OWiG auch dazu bestimmt sei, im Interesse der Markteilnehmer das Marktverhalten zu regeln. Im Ergebnis verneinte das Gericht aber den Unterlassungsanspruch der Kläger, da die veröffentlichten Inserate nicht gegen $\S 119$ Abs. 1 und $\S 120$ Abs. 1 Nr. 2 OWiG verstößen.

Die hier zunächst vorrangig in Betracht zu ziehende Vorschrift des $\S 119$ Abs. 1 Nr. 2 $\mathrm{OWiG}^{2}$ verbietet das Anbieten, Ankündigen, Anpreisen und die Bekanntgabe von Erklärungen solchen Inhalts, die sich auf Gelegenheiten zu sexuellen Handlungen beziehen, wenn dies in grob anstößiger Weise durch Verbreitung von Schriften, Ton- oder Bildträgern, Abbildungen oder Darstellungen oder durch das öffentliche Zugänglichmachen von Datenspeichern geschieht. Einen solchen Verstoß lehnte der BGH hier (ohne nähere Ausführungen) schon zu Recht damit ab, dass die Werbung durch die Inserate nicht in grob anstößiger Weise erfolgte. Eine grob anstößige Weise wird angenommen, wenn entweder der Inhalt oder die Art der Verbreitung in erheblicher Weise Sitte, Anstand und Schamgefühl verletzen ${ }^{3}$ bzw. die Angebote derart aufdringlich sind, dass das nach Auffassung der Mehrheit der
Bevölkerung gebotene Maß an Zurückhaltung überschritten ist. ${ }^{4}$ Die beanstandeten Inserate waren mehr oder weniger diskret („Erotik Massage“, „Süßer Engel“, ,Jung und Wild“, „Süße Lisa (18) verwöhnt dich heiß"), entsprechen inhaltlich jedenfalls dem, was sich in den überregionalen Boulevardzeitungen täglich finden lässt. Dabei kann, von Ausnahmen abgesehen, davon ausgegangen werden, dass sich der allergrößte Teil der Bevölkerung, nicht zuletzt auch aufgrund eines Wandels moralischer Vorstellungen, daran nicht (mehr) stößt.

Im Anschluss prüfte der BGH einen Verstoß gegen $\S 120$ Abs. 1 Nr. 2 OWiG. Danach handelt ordnungswidrig, wer durch das Verbreiten von Schriften, Ton- oder Bildträgern, Datenspeichern, Abbildungen oder Darstellungen Gelegenheit zu entgeltlichen sexuellen Handlungen anbietet, ankündigt, anpreist oder Erklärungen solchen Inhalts bekannt gibt. Die Vorschrift verbietet daher dem Wortlaut nach jede Werbung für entgeltliche sexuelle Kontakte. Im Gegensatz zu $\S 119$ Abs. 1 Nr. 2 OWiG ist es nicht erforderlich, dass die Werbung in grob anstößiger Weise erfolgt.

So wurde die Vorschrift auch nach bislang ganz überwiegender Ansicht als abstraktes Gefährdungsdelikt verstanden, vornehmlich zum Schutz der Allgemeinheit, namentlich von Jugendlichen, vor den mit der Prostitution generell verbundenen Belästigungen und Gefahren. ${ }^{5}$

Auf eine konkrete Belästigung oder Gefährdung im Einzelfall kam es nicht an, so dass auch die Werbung in sachlicher, nüchterner oder verdeckter Form der Vorschrift unterfiel. Entscheidend war allein dass sich der objektiv erkennbare Inhalt der Werbung auf Prostitution bezieht. ${ }^{6}$ Danach wären die beanstandeten Anzeigen ohne weiteres unter das Werbeverbot subsumierbar gewesen. Dieser Ansicht ist der BGH in seinem Urteil nun entgegengetreten, indem der Senat das Verbot von Werbung für entgeltliche sexuelle Kontakte auf die Fälle beschränkt sehen will, in denen durch die Werbung eine konkrete Beeinträchtigung von Rechtsgütern der Allgemeinheit, insbesondere des Jugendschutzes, eintritt. ${ }^{7}$ Dies sei der Fall, wenn die Werbung nach Aufmachung, Inhalt oder 
Umfang nicht in der gebotenen zurückhaltenden Form erfolgt oder nach Art des Werbeträgers und seiner Verbreitung geeignet ist, die schützbedürftigen Rechtsgüter zu gefährden, wobei nicht erforderlich ist, dass die weitergehenden Erfordernisse des § 119 Abs. 1 erreicht sind. ${ }^{8}$ An diesem Maßstab gemessen, vermochte das Gericht keinen Verstoß gegen $\S 120$ Abs. $1 \mathrm{Nr}$. OWiG durch die Veröffentlichung der Inserate festzustellen. ${ }^{9}$

Zur Begründung seiner einschränkenden Auslegung von § 120 Abs, 1 Nr. 2 OWiG greift der Senat auf das ProstG zurück, durch das der Gesetzgeber einem Wandel in weiten Teilen der Bevölkerung Rechnung getragen habe, die Prostitution nicht mehr als "schlechthin als sittenwidrig“ anzusehen. ${ }^{10}$ Soweit erkennbar ist das Urteil damit die erste höchstrichterliche Rechtsprechung aus der klar hervorgeht, dass das ProstG bei der Auslegung von Vorschriften aus anderen Rechtsgebieten in diesem Sinne zu berücksichtigen ist. Dieses scheint zwar bei einem solchen Verständnis des ProstG auf der Hand zu liegen, ${ }^{11}$ hat sich aber bislang bei weitem nicht durchgesetzt. Bereits bei der Beurteilung darüber, ob Verträge über entgeltliche sexuelle Handlungen nun nicht mehr „sittenwidrig“ sind, herrscht Uneinigkeit. So geht die ganz überwiegende Ansicht nach wie vor von der Sittenwidrigkeit im Sinne von $\S 138$ BGB und damit von der anfänglichen Nichtigkeit solcher Verträge aus, sie werden nur nachträglich teilweise wirksam. ${ }^{12}$ Und so herrscht auch eine erhebliche Unsicherheit im Umgang mit rechtlichen Fragen außerhalb des unmittelbaren Geltungsbereiches des Prostitutionsgesetzes, etwa im Gewerbe-, Gaststätten und Bauordnungsrecht. Gerade im Baurecht hat das ProstG nicht zu einer neuen rechtlichen Behandlung oder gar planungsrechtlichen Gleichstellungen für Bordelle, bordellartige Betriebe oder der Wohnungsprostitution mit anderer legaler Gewerbeausübung geführt; eine Ausstrahlungswirkung des ProstG wird für diesen Bereich weitestgehend verneint. ${ }^{13}$

Dies gilt ebenso für das Strafrecht. Ein Gesetzesentwurf des Bundesrates betreffend die Einführung eines Tatbestandes zur Bestrafung von Freiern von Menschenhandelsopfern, sieht auch eine Revision der mit dem ProstG geänderten bzw. abgeschafften Tatbestände der Förderung der Prostitution (§ 180a StGB) sowie der dirigistischen $\mathrm{Zu}$ hälterei (181a Abs. 2 StGB) vor. ${ }^{14}$ Bei den im Jahre 2005 neu gefassten Straftatbeständen zum Menschenhandel wurden Wertungswidersprüche zum ProstG bewusst hingenommen, in dem die dort getroffenen zivilrechtlichen Entscheidungen keine Berücksichtigung fanden. ${ }^{15}$

Ein Gesetz zur Regelung der rechtlichen Stellung von Prostituierten nützt nichts, wenn die freie Berufausübung der in diesem Sektor tätigen Personen über den Umweg des Straf- oder Ordnungsrechtes nach wie vor erschwert oder gar noch schwerer gemacht wird. Es ist daher noch ein weiter Weg - und das knapp fünf Jahre nach Inkrafttreten des ProstG - bis zur Beseitigung der rechtlichen Diskriminierung der Prostitution. Von daher ist das Urteil des BGH als Schritt in die richtige Richtung ausdrücklich zu begrüßen.

\section{Martin Schaar ist wiss. Mitarbeiter im ISK-Kiel.}

\section{Literatur:}

Erbs, Georg (Begr.)/ Kolhaas, Max/ Ambs, Friedrich (Hg.);

Strafrechtliche Nebengesetze, Band III, München, Stand: 163. Lieferung, Sept. 2006 (zit.: Erbs/Koolhass-Bearbeiter).

Frommel, Monika/ Schaar, Martin

Einwände gegen den am 19.02.2005 neu gefassten Straftatbestand des Menschenhandels in § 232 Abs, 1, NK 2005, S. 61-63.

von Galen, Margaräte Gräfin,

Rechtsfragen der Prostitution: Das Prostitutionsgesetz und seine Auswirkungen, München, 2004.

Malkmus, Katrin,

Prostitution in Recht und Gesellschaft, Frankfurt a. M., 2005.

Palandt, Otto,

Bürgerliches Gesetzbuch, 65. Aufl., München

2006, (zit.: Palandt-Bearbeiter).

Senge, Lothar, (Hg.),
Karlsruher Kommentar zum Gesetz über Ordnungswidrigkeiten, 3. Aufl., München, 2006 (zit.: KK-OwiG-Bearbeiter).

Stühler, Hans-Ulrich

Auswirkungen des Prostitutionsgesetzes auf das Bau-, Gaststätten- und Gewerberecht, GewArch, 2005, S. 129-132.

Rebmann, Kurt/ Säcker, Franz Jürgen/ Rixecker, Roland (Hg.)

Münchener Kommentar zum Bürgerlichen Gestetzbuch, Band 1a, Allgemeiner Teil (Auszug), 4. Aufl. 2003 (zit.: MüKo-Bearbeiter).

\section{Fußnoten:}

1 Gesetz zur Regelung der Rechtsverhältnisse der Prostituierten, BGBl. 2001, I. S. 3983.

$2 \S 119$ Abs. 1 Nr. 2 OWiG ist im Verhältnis zu Abs. 1 Nr. 1eine Sonderregelung, die sich insbesondere gegen Anzeigen, in denen Angebote zu sexuellen Kontakten gemacht werden, wendet (BT-Drs. VI/1552, S.37). Sie verdrängt bei Vorliegen auch § 120 Abs. 1 Nr. 2 OWiG (Erbs/Kohlhaas-Senge, § 119, Rn. 22).

3 BT-Drs. VI/3521 S. $62 \mathrm{ff}$.

4 KK-OWiG-Kurz, § 119, Rn. 15.

5 OLG Karlsruhe NJW 1978, 61; KK-OWiG-Kurz, § 120 OWiG, Rn. 2,3; Erbs/Kohlhaas-Senge, § 120 OWiG, Rn. 1.

6 BGH NJW 1992, S. 2557, 2558 mit weiteren Nachweisen.

7 BGH Urt. v. 13.07.2006 - I ZR 241/03, Rn. 25.

8 BGH Urt. v. 13.07.2006 - I ZR 241/03, Rn. 26.

9 Dies entspricht im Ergebnis wohl auch der gängigen Praxis. Wie eine Nachfrage bei einer überregional, täglich erscheinenden Zeitung, die derartige Inserate veröffentlicht ergab, werden Anzeigen anhand einer "Negativliste" mit bestimmten Begriffen geprüft und gegebenenfalls nicht veröffentlicht. Ein Bußgeldverfahren wegen Verstoßes gegen §§ 119 Abs. 1, 120 Abs. 1 Nr. 2 OWiG hatte es gegen den angefragten Verlag in den letzten Jahren nicht gegeben.

10 BGH Urt. v. 13.07.2006 - I ZR 241/03, Rn. 24.

11 Hierzu grundlegend von Galen, S. 101ff.

12 Vgl. OLG Schleswig, NJW 2005, S. 225, 227; Palandt-Heinrichs, Anhang zu § 138, Rn. 2 mit weiteren Nachweisen; a.A. MüKo-Armbrüster, ProstG, § 1, Rn. 8, Malkmus, S. 79 und 151.

13 Vgl. Stühler, GewArch 2005, S. 129, 132.

14 BT-Drs. 16/1343.

15 Frommel/Schaar, NK 2 (2005). 